\title{
Why nanodiamond carriers manage to overcome drug resistance in cancer
}

\author{
Veronika Benson', Abbas Amini' ${ }^{2,3}$ \\ IInstitute of Microbiology, Czech Academy of Sciences, Prague 14220, Czech Republic. \\ ${ }^{2}$ Department of Mechanical Engineering, Australian College of Kuwait, Safat 13015, Kuwait. \\ ${ }^{3}$ Center for Infrastructure Engineering, Western Sydney University, Penrith, NSW 2751, Australia.
}

Correspondence to: Dr. Veronika Benson, Institute of Microbiology, Czech Academy of Sciences, Vídeňská 1083, Prague 14220, Czech Republic. E-mail: benson@biomed.cas.cz

How to cite this article: Benson $\mathrm{V}$, Amini A. Why nanodiamond carriers manage to overcome drug resistance in cancer. Cancer Drug Resist 2020;3:854-66. http://dx.doi.org/10.20517/cdr.2020.52

Received: 21 Jul 2020 First Decision: 12 Aug 2020 Revised: 1 Sep 2020 Accepted: 14 Sep 2020 Available online: 12 Oct 2020

Academic Editor: Vladimir P. Torchilin Copy Editor: Cai-Hong Wang Production Editor: Jing Yu

\begin{abstract}
Nanodiamonds represent an attractive potential carrier for anticancer drugs. The main advantages of nanodiamond particles with respect to medical applications are their high compatibility with non-cancerous cells, feasible surface decoration with therapeutic and cancer-cell targeting molecules, and their relatively low manufacturing cost. Additionally, nanodiamond carriers significantly increase treatment efficacy of the loaded drug, so anticancer drugs execute more effectively at a lower dose. Subsequently, lower drug dose results in less extensive side effects. The carriers decorated with a targeting molecule accumulate primarily in the tumor tissue, and those nanodiamond particles impair efflux of the drug from cancer cells. Therapeutic approaches considering nanodiamond carriers were already tested in vitro, as well as in vivo. Now, researchers focus particularly on the possible side effects of nanodiamond carriers applied systemically in vivo. The behavior of nanodiamond carriers depends heavily on their surface coatings, so each therapeutic complex must be evaluated separately. Generally, it seems that site-specific application of nanodiamond carriers is a rather safe therapeutic approach, but intravenous application needs further study. The benefits of nanodiamond carriers are remarkable and represent a potent approach to overcome the drug resistance of many cancers.
\end{abstract}

Keywords: Nanodiamond, drug carrier, drug resistance, cancer therapy, nanoparticles 


\section{INTRODUCTION}

Cancer resistance represents a major cause in cancer treatment failure. During cancer development, cancer cells adapt different strategies to avoid the toxicity of anticancer drugs including fast drug efflux. Increased efflux eliminates the possibility of actual drug action and eventually, it decreases retention of the drug within the tumor. Then, the excluded drugs are quickly cleared from the organism. Application of a higher drug dose is often associated with undesired serious side effects without real therapeutic benefits. Compared to specialized cancer cells, cancer stem cells (CSC) are highly resistant to any therapy due to their nature and quiescent state. Until now, various nanomaterials have been developed with the intention of increasing the therapeutic efficacy of cancer treatment. Nanomaterials were studied in vitro and in vivo, and some reached clinical trials. Those include poly (lactic-co-glycolic acid) (PLGA) nanoparticles, metallic nanoparticles, carbon nanotubes and nanoparticles, polymer- and lipid-based materials, and many others $^{[1-4]}$. Generally, it seems that the involvement of nanoparticles in designed drug formulae could be indeed beneficial to fight resistant cancer (and CSC) cells. In this review, we focus on carbon nanoparticles, specifically nanodiamonds, and their capacity to improve drug efficacy with respect to treatment-resistant cancer cells.

\section{DRUG NANOCARRIERS}

Nanocarriers can significantly improve the efficacy of cancer treatment by overcoming low retention of anti-cancer drugs in tumor tissue and fast drug clearance from circulation. Additionally, nanocarriers also exhibit decreased off-target toxicity in comparison with free drugs. So far, it seems that interactions of nanoparticles with immune cells (excluding the monocyte/macrophage lineage) are $\operatorname{rare}^{[5-6]}$. Among other nanomaterial that were developed for biomedical use, carbon material (nanotubes, graphene, fullerenes, carbon dots, films), and particularly nanodiamonds caught scientists' attention due to their potential use in drug delivery and bioimaging ${ }^{[5,7-13]}$.

\section{NANODIAMONDS}

\section{Nanodiamond synthesis}

Nanodiamonds (NDs) represent a heterogeneous family of nanoparticles in terms of size, shape, or surface potential. Those properties arise primarily from different means of preparation, and they significantly affect nanodiamond particle properties and their optimal final use. The main approaches to obtain nanodiamonds for biomedical use are via detonation [detonation ND (DND)] and growth under high-pressure hightemperature $(\mathrm{HPHT})^{[14]}$. While detonation nanodiamonds are mostly of smaller size (1-10 nm), a positive surface charge, and higher sp2 content; the HPHT particles are larger (35-100 nm), negatively or positively charged, and have a lower sp2 content ${ }^{[15]}$. In addition, HPHT nanodiamonds undergo a milling procedure that equips them with sharp edges instead of a spherical shape. Finally, the HPHT particles are large enough to possess luminescent center(s). The luminescent centers are nitrogen-vacancy centers introduced into milled HPHT nanodiamonds by high-energy irradiation ${ }^{[16]}$.

\section{Nanodiamond properties and benefits}

The size of the nanodiamond influences its circulation time in the blood stream and its accumulation in particular sites. Depending on their final use, we can benefit from small or large particles. Small particles $5 \mathrm{~nm}$ in diameter are cleared quickly by the kidneys. Their circulation time in peripheral blood is too short to be accumulated in reticuloendothelial system (RES, particularly liver, kidney, lymph nodes, and spleen) and too short to be effectively accumulated in tumor sites ${ }^{[17]}$. In tumors, the nanodiamonds primarily accumulate via enhanced permeability retention (EPR) or via targeted homing, based on the tumor-specific structure involved in nanodiamond coating ${ }^{[18,19]}$. Larger particles (about $50 \mathrm{~nm}$ ) cannot be cleared by the kidneys and stay in circulation for long periods. They eventually accumulate in tumor tissue, but they can also accumulate in $\mathrm{RES}^{[17]}$. There are reports describing that even though nanodiamonds accumulated at any 


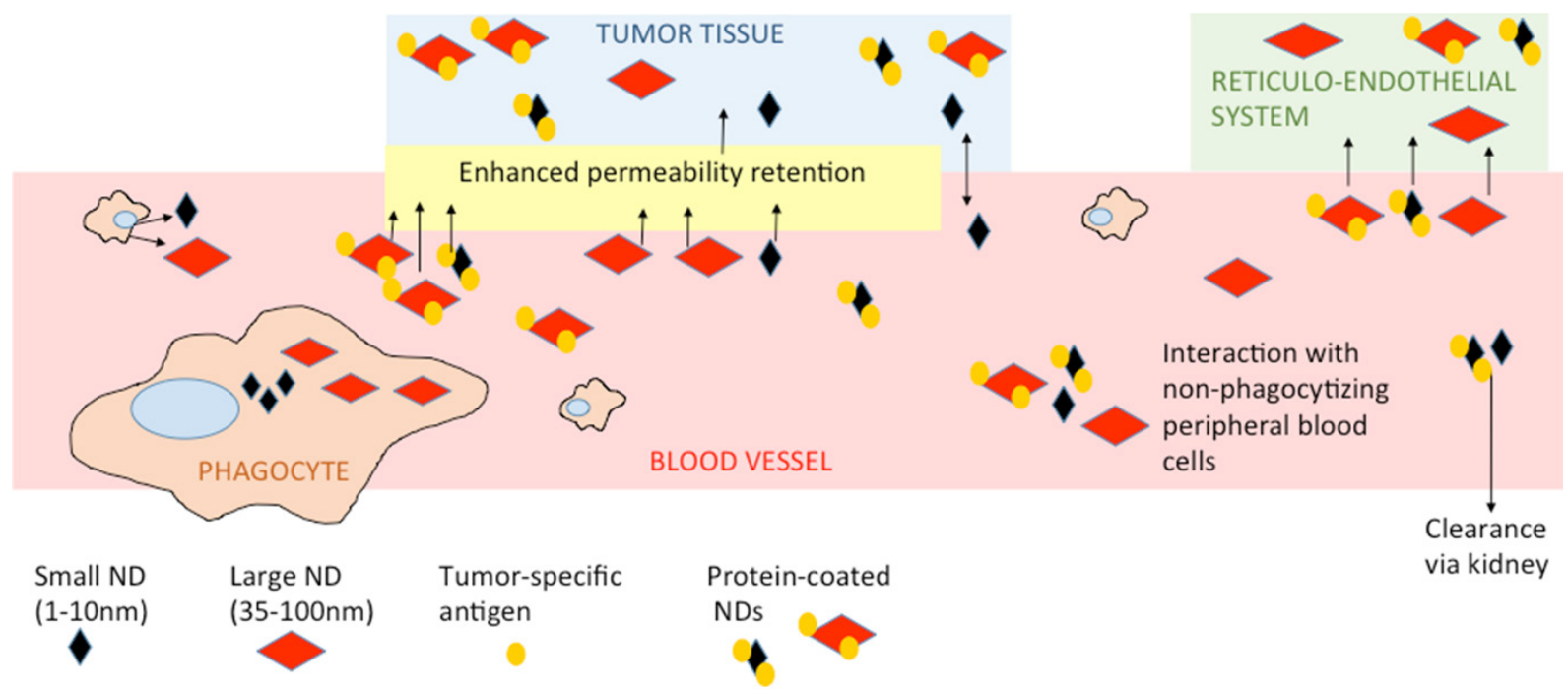

Figure 1. Fate and basic interactions of nanodiamonds after their intravenous administration. The differences between shielded vs. unshielded and small vs. large nanoparticles are shown. ND: nanodiamond

RES site, they did not cause any substantial harm at the organ or organism levels ${ }^{[20,21]}$. However, there are questions on the accuracy of that statement, such as whether there are any unpublished data contradicting that statement; for example, after using differently prepared or modified carriers that failed in toxicity assays or exhibited any other unfavorable effects. Also, as nano-bio research progresses, we learn more about the potential, pitfalls, and modifications of nanocarriers. A big question in early research was the formation of nanodiamond aggregates, particularly after their intravenous application. Nowadays, different surface coatings or space stabilization have solved undesired aggregation ${ }^{[10,22]}$. The optimal approach seems to be coating with proteins that keep particles relatively dispersed and protect them from excessive protein corona formation. Protein corona forms on the surface of unshielded nanodiamonds after exposure to peripheral blood, or to a lesser extent, after exposure to serum-supplemented culture media ${ }^{[23]}$. The protein structure added as part of the nanodiamond coating will also shield nanodiamond complexes from uptake by peripheral macrophages ${ }^{[5]}$. Conveniently, if we coat nanodiamonds with a tumor-specific antibody, the nanodiamond will effectively accumulate at the tumor site ${ }^{[5,24]}$. Simplified, the fate of nanodiamond carriers after systemic application is shown in Figure 1.

Surprisingly, nanodiamond aggregation was found to be beneficial concerning cancer resistance. Here, controlled agglomeration of nanoparticles overcame the mechanism of tumor drug resistance ${ }^{[25,26]}$.

One of the failure points in drug delivery is the inability of drug carrier to escape from the endosome into the cytoplasm after intake by a cancer cell. The HPHT nanodiamond carriers effectively delivered their cargo into the cell cytoplasm. Chu et al. ${ }^{[27]}$ suggested that nanodiamonds were successful in escaping endosomes because they possessed sharp edges, and potentially positive surface charge. After unloading cargo within the endosome, both the sharp edges and the positive charge of the nanodiamonds destabilize the endosomal membrane, enabling the escape of the nanodiamonds and their cargo into the cytoplasm ${ }^{[10]}$. Subsequently, the nanodiamonds usually accumulate in the cytoplasm, close to the nuclear envelope, but rarely enter the nuclei ${ }^{[8,10,11,28-30]}$. There is a recent report regarding the detection of nanodiamonds in nuclei ${ }^{[31]}$; however, it is more likely that the nanodiamonds were embedded within the nuclear membrane as described by Gismondi et al. ${ }^{[32]}$. A different situation may occur in the case of coated nanodiamonds. For example, Martín et al. ${ }^{[33]}$ proposed that fenton-treated nanodiamonds were able to enter directly into cell nuclei. 
The shape of the nanodiamond significantly affects its internalization and accumulation within the cell. We have discussed that sharp edges enable the nanodiamond to escape into the cytoplasm and accumulate there. On the other hand, if the particle edges are less sharp or the nanodiamond is spherical, then the carrier resides in the endosome until it matures to a lysosome, and subsequently the nanodiamond will be expelled from the cell via exocytosis ${ }^{[27]}$.

Different methods of nanodiamond preparation result in particle with positive or negative surface charge. According to their subsequent use, each particle is modified, and total surface charge changes. Positively charged particles can be directly linked with negatively charged drugs or macromolecules. The nanodiamond exhibiting a total negative charge is often coated with a positively charged polymer that enables electrostatic binding of an anionic drug/biomolecule, and the cationic polymer promotes nanocarrier entry into cells ${ }^{[34]}$.

Concerning cell compatibility, one of the key parameters is the amount of sp2 conformation. The level of sp2 contamination depends heavily on the preparation method, and it decreases by optional nanoparticle oxidation. In contrast to sp3, sp2 conformation is less cell compatible. And it has been the probable cause of lower cell-compatibility of detonation nanodiamonds ${ }^{[15]}$. Detonation nanodiamonds contain a high amount of sp2 on their surface due to their preparation method.

Next to specific characteristics resulting from preparation procedure, several properties are common to most nanodiamond carriers. The nanodiamonds are chemically stable, possess rigid structures with octahedral symmetry, and have a large surface area. Regarding sustainable production, their fabrication is relatively low cost and scalable ${ }^{[28]}$.

There are remarkable advantages of nanodiamond particles over other available organic or inorganic carriers. Mainly, nanodiamonds are very flexible regarding surface decoration, and nanodiamonds with luminescent centers are easily traceable. Specifically, surface versatility makes them superior even to synthetic polymeric nanocarriers such as PLGA particles that have already used for drug delivery in clinical application ${ }^{[4]}$. The final, yet important benefit of nanodiamonds is their great compatibility with different live objects. Both naked and coated nanodiamonds were well tolerated by many different cell types including immune cells and specialized tissue cells ${ }^{[29,30]}$. No threatening side effects were found even after long-term persistency of naked nanodiamonds within the body ${ }^{[21]}$. We and others ${ }^{[5,35]}$ have found interactions of coated nanodiamonds with specialized cells, but they did not seem to promote any pathology. It is important to mention though that the interaction of nanoparticles (including nanodiamonds) with specialized cell types are still under extensive research. As the research community witnesses the huge potential of nanocarriers particularly for anticancer therapy, maintaining high scrutiny over these nanocarriers is very important. However, for the safe use of nanocarriers, we need more information regarding particular carrier types and their surface modifications.

Unfortunately, the reported data are rather heterogeneous and represent a significant issue for comparing the behavior of nanodiamond carriers. Nanodiamond characterization parameters and toxicity evaluation methods are not united, and there remains a lack of important information (for example size, method of preparation, and charge) in many reports. Generalized interpretation of such outputs is complicated, because as discussed earlier, size, shape, surface charge, method of preparation, method of coating, and type of coating all affect the final behavior and nano-bio interaction of the carriers.

\section{Nanodiamonds as drug carriers}

Nanodiamonds have found use in many technological approaches and biomedical areas including imaging, microbial resistance management, bone tissue engineering, and root canal fillings ${ }^{[15,36]}$. Overall, their 
application as drug carriers is the most prevalent. The main reason is that nanodiamonds can be easily functionalized to deliver a wide range of therapeutics and target specific cancer cells. In parallel, concerning acute toxicity, most nanodiamond-based complexes are well tolerated ${ }^{[9,10,20,29,30]}$. The effectiveness of drug adsorption and desorption on/from nano-carriers depends on diamond core purity, surface chemistry, and dispersion quality, as well as on environmental factors - ionic composition, $\mathrm{pH}$, and temperature ${ }^{[12]}$.

As discussed earlier, the nanodiamond surface can be decorated with different functional groups enabling interactions with water molecules or biologically relevant conjugates ${ }^{[4]}$. Nanodiamonds carrying anticancer drugs promote drug accumulation at the tumor site by passive or active mechanisms. The passive mechanism of drug accumulation is based on enhanced permeability and retention effect due to undeveloped tumor vasculature ${ }^{[37]}$. The larger size of carrier-drug is advantageous for the drug accumulation. The nanodiamond carriers possessing a targeting moiety on their surface promote drug accumulation due to active targeting of tumor-specific antigens ${ }^{[38]}$.

Anticancer drugs often linked to the nanodiamond surface are anthracyclines (specifically doxorubicin), daunorubicin, and epirubicin. Anthracyclines are DNA intercalating agents, exhibiting high effectivity in tumor growth suppression; however, they are also extremely toxic. Their major limitation is dose-dependent side effects such as myelosuppression, cardiotoxicity, and the development of acute myeloid leukemia ${ }^{[39]}$. Many reports have shown that the binding of anthracyclines to the nanodiamond carriers significantly reduced the effective dosage, resulting in lesser side effects ${ }^{[40-44]}$. The first reports describing transportation of doxorubicin by nanodiamonds used drug physisorption onto nanoparticles. That approach enabled the easy binding and release of the drug without any chemical modification or active targeting ${ }^{[45,46]}$. Later on, Moore et al. ${ }^{[42]}$ and Zhang et al. ${ }^{[4]}$ decorated the nanodiamond surface with antibodies recognizing epidermal growth factor receptor (EGFR) on the surface of breast cancer cells. That modification promoted targeted delivery of doxorubicin-nanodiamond complexes into EGFR positive cancer cells.

Since the anthracyclines administered alone triggered serious side effects, the researchers focused also on the premature release of the drug from the nanodiamond core in response to different ambiances ${ }^{[43]}$. Especially important was the complex stability after exposure to sera proteins because early drug release in peripheral blood could trigger the above-mentioned toxicity, similar to the free drug. Wang et al. ${ }^{[43]}$ studied epirubicin release from nanodiamond carriers after exposure to sera proteins under physiological $\mathrm{pH}$ of blood. They found no epirubicin release to sera up to six hours after application. After cancer cells internalized the complexes, acidic intracellular ambiance, together with intracellular proteins promoted the release of the drug from the nanodiamond core.

Lin et al ${ }^{[41]}$ reported another interesting study that used EGFR-specific monoclonal antibody cetuximab as a targeting molecule. That antibody decorated nanodiamonds delivered paclitaxel into human colon cancer cells in vivo (xenograft in nude mice). Paclitaxel is a microtubule inhibitor and its delivery into cancer cells induced mitotic catastrophe and reduced tumor growth ${ }^{[41]}$. Until now, studies covering nanodiamondmediated drug delivery focused on the efficacy of drug accumulation in tumors or RES, on tumor growth, and sometimes on acute organ toxicity. However, there are only a few studies concerning non-tumoral cells, for example, those residing in the peripheral blood. Those cells are likely to interact to some degree with the applied nanodiamond-drug complexes. Even though the decoration of nanodiamond surfaces with proteins shielded complexes from being engulfed my macrophages ${ }^{[5]}$, some carrier formulations could interact with red blood cells ${ }^{[35]}$ or granulocytes ${ }^{[5]}$. Here we mention exemplary reports describing direct or indirect effects of nanodiamond-drug carriers on different blood elements. Madamsetty et al. ${ }^{[48]}$ conducted a complex study of pancreatic ductal adenocarcinoma treatment using nanodiamond cores coated with poly (ethylene glycol) (PEG) and doxorubicin. The authors used a type of pancreatic cancer lacking effective therapy as their model. They showed that doxorubicin carried by a nanodiamond 
core was more effective than the free drug while exhibiting lower side effects. The complexes triggered reduced tumor growth and accumulated in the tumor site. The authors then considered the interaction of nanocomplexes with blood macrophages. The coated nanodiamond complexes exhibited no toxic effect on the employed macrophages. Unfortunately, the research engaged leukemia cell line PLB-985 instead of the primary culture derived from tissue or peripheral macrophages. Thus, the effect of the nanodiamondcomplex on macrophages differs from the in vivo situation and may be a concern due to PEG-mediated hypersensitivity ${ }^{[49]}$. Křivohlavá et al. ${ }^{[5]}$ used an alternative approach; here, the studied complex contained a nanodiamond core, poly (ethylene imine), interfering RNA, and transferrin as a tumor-specific marker. The authors showed effective accumulation of loaded carriers in tumors after systemic administration followed by the specific knockdown of oncogenic microRNA-135b in tumor cells. The nanodiamond-mediated side effects were evaluated ex vivo in aspirates of peripheral blood cells and peritoneal macrophages. After systemic application, plasma samples and tissues were tested as well. In summary, the authors did not confirm any significant toxicity on macrophages, but they pointed out the possible interaction of coated nanodiamonds with granulocytes and splenocytes ${ }^{[5]}$.

It is worth mentioning that not only nanodiamond particles but also nanodiamond scaffolds found application as drug carriers. For example, Suliman et al ${ }^{[50]}$ described the successful delivery and release of bone morphogenetic protein-2 by nanodiamond-based bone transplants.

Remarkably, the nanodiamond-based drug carriers have also been included in the Phenotypic Personalized Medicine-Drug Development (PPM-DD) approach. That platform focuses on de-risked drug development due to the systematic rational design of optimal therapeutic combinations. The PPM-DD platform allows rapid determination of optimal drug combinations and includes both conventional as well as nanocarrierbased approaches ${ }^{[4]}$.

In addition to drug delivery, nanodiamond particles also served in cancer cell imaging. As an example, conjugates of nanodiamond particles with gadolinium (III) were used for magnetic resonance ${ }^{[51]}$. Here, the nanodiamond-gadolinium conjugates increased sensitivity of magnetic resonance in contrast to gadolinium alone. At the same time, using those conjugates enabled lowering the sufficient dosage of gadolinium as well. In addition to improvements in magnetic resonance, nanodiamonds assisted in the detection of stem cells. Wu et al.$^{[52]}$ used fluorescent nanodiamonds to label lung stem cells in order to track their engraftment and distribution during the regeneration of lung tissue after injury (murine model).

\section{OVERCOMING DRUG RESISTANCE BY NANODIAMOND CARRIERS}

\section{Mechanisms of anti-cancer drug resistance}

Treatment resistance to conventional chemotherapy and radiotherapy is a key property of cancer cells that enables their escape and cancer treatment failure ${ }^{[53]}$. Mechanisms of drug resistance differ and encompass the quiescent state ${ }^{[54]}$, fast drug efflux from the cell ${ }^{[55]}$, drug inactivation, mutation of the drug target, enhanced DNA damage repair ${ }^{[56]}$, inhibition of cell death ${ }^{[57]}$, epigenetics, or epithelial-mesenchymal transition ${ }^{[58]}$. The contribution of tumor environment, especially stromal cells, is also important ${ }^{[59]}$.

Drug uptake and drug efflux is regulated mainly by ATP-binding cassette (ABC) protein transporters, including P-glycoprotein (MDR1 or $\mathrm{ABCB} 1$ ). This molecule has been extensively studied due to its fundamental role in multidrug resistance. Overexpression of the $\mathrm{ABC}$ transporters correlates with poorer drug response and poorer clinical prognosis ${ }^{[60]}$. The chemoresistance mediated by $\mathrm{ABC}$ transporters has been often associated with cancer stem cells, and is likely one of the major mechanisms, why cancer stem cells escape conventional therapy and trigger tumor recurrence ${ }^{[6]]}$. Therefore, there is a substantial need to develop new strategies to overcome drug resistance and target cancer stem cells. In some reports, researchers tested targeting of cancer stem cells via stem cells-specific markers such as CD44+, CD90+, 


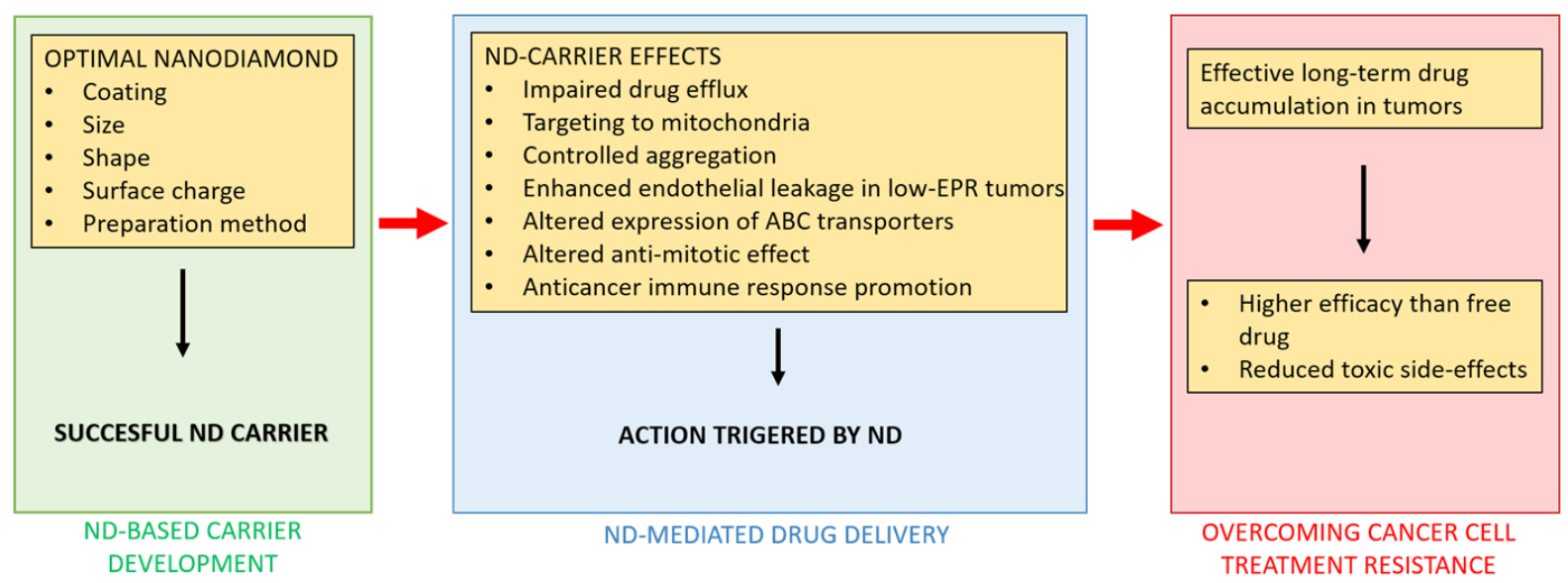

Figure 2. Summary of nanodiamond (ND)-mediated actions that contribute to overcome the treatment resistance of cancer cells

CD $133+{ }^{[62]}$, or specific signaling pathways like Notch, Hedgehog, or transforming growth factor- $\beta^{[63]}$. Recently, the application of nanomaterials has gained additional attention because it offers targeted, controlled, and effective drug delivery and release.

\section{Nanodiamonds against drug resistant cancer}

Nanodiamond particles employed as drug carriers possess several properties that help to overcome resistance of cancer cells to conventional therapy. The summarized mechanisms of nanodiamond-triggered cancer cell response are shown in Figure 2.

First, nanodiamonds impair drug efflux from target cells. Cellular transport proteins that pump drugs out of the cells cannot recognize and carry the truncated octahedral structure of the nanodiamond ${ }^{[40]}$. Therefore, the carrier-drug complexes stay inside the cells. Thus, accumulation of the drug inside the cancer cell increases drug efficacy on cancer cells. Simultaneously, drug retention inside cancer cells exposes the nanodiamond-drug complex to the acidic ambiance (low $\mathrm{pH}$ ) inside the cells that promotes the release of the drug from the carrier. Accumulation and release of the drug inside cancer cells also reduces side effects of drugs on the surrounding tissue ${ }^{[64]}$. For example, Wang et al ${ }^{[43]}$ employed epirubicin linked to $5 \mathrm{~nm}$ nanodiamond cores. This complex effectively targeted not only cancer cells, but also cancer stem cells, and thus nanodiamond-based treatment prevented secondary tumor formation in a liver cancer xenograft model. Importantly, the study showed that if linked to nanodiamonds, the epirubicin could be used also in otherwise lethal doses. That means the developed drug formula could be used in patients who could not tolerate the conventional free drug due to its toxic side effects.

Second, the efflux of the anticancer drug by the tumor cells was also bypassed by targeting the loaded nanodiamond carrier all the way to mitochondria via mitochondrial leader sequences (MLS peptide) ${ }^{[65]}$. Chan et al. ${ }^{[65]}$ employed fluorescent nanodiamonds loaded with doxorubicin that were simultaneously coated with cancer targeting structures (PEGylated folic acid) and mitochondria targeting MLS peptides. Following endosomal escape, the MLS peptide facilitated transportation of the therapeutic complex directly into mitochondria where doxorubicin induced programmed cell death.

Third, as we have already discussed, nanodiamond size or even the controlled aggregation of nanodiamondbased complexes can be beneficial. The final size of the complex, containing at least a nanodiamond core and drug, usually exceeds the limits for renal clearance, and the loaded nanodiamonds circulate in the peripheral blood for a prolonged time. Increased half-time of nanodiamond-drug circulation maximizes accumulation of the therapeutic complexes within the tumor via an enhanced permeability retention effect, 
resulting in constant tumor exposure to the $\mathrm{drug}^{[40,66]}$. Chang et al ${ }^{[25]}$ were the first to clearly show that selfaggregation of detonation nanodiamond particles significantly contributed to the enhanced therapy efficacy of drug-resistant tumors.

Detonation nanodiamonds were linked with anthracyclines, and their aggregates reached average sizes about $80 \mathrm{~nm}$ in diameter. Those aggregates appeared to be critically important for amended tumor therapy. They increased circulatory half-life of the drug-carrier 10-fold, resulting in improved intratumoral drug retention ${ }^{[25,26]}$. Similarly, Toh et al. ${ }^{[67]}$ showed enhanced retention of nanodiamond-linked mitoxantrone in chemoresistant breast cancer cells.

Fourth, nanodiamond carriers increase intracellular levels of reactive oxygen species and calcium $\left(\mathrm{Ca}^{2+}\right)$, resulting in enhancement of endothelial leakage. That phenomenon could be used in low-EPR tumors that exhibit reduced EPR-based accumulation of drugs. The nanodiamond carriers encourage permeation of vascular endothelium by the anticancer drug and enable the drug to reach target tumor tissue ${ }^{[68]}$.

Fifth, nanodiamond-anthracyclin complexes altered the expression of the protein transporters responsible for drug efflux. Specifically, nanodiamond cores linked with doxorubicin and PEG decreased the expression of ABCG2, a member of the ABC transporter family ${ }^{[48]}$. The ability of nanodiamond carriers to effectively deliver anthracyclines into cancer cells and overcome their immediate efflux promises a suitable delivery platform to treat cancer stem cells ${ }^{[43,67]}$.

Sixth, nanodiamond carriers altered the anti-mitotic effect of loaded drug citropten. Citropten is a natural compound found in citruses, and free drug application leads to apoptotic cell death of cancer cells. On the other hand, citropten loaded onto nanodiamond carriers interferes with the actin filaments involved in mitosis $^{[32]}$. Therefore, the citropten-nanodiamond complexes inhibited proliferation of rapidly dividing cancer cells but exhibited minimal toxic effect on healthy tissues. Lowering of unfavorable side-toxicity by linking certain drugs to a nanodiamond carrier was discussed above in the case of anthracycline. It could be a useful therapeutic approach in fast growing tumors even though it does not solve the existence of quiescent cancer stem cells.

Seventh, the nanodiamond carriers promoted an anticancer immune response. On this topic, Yuan et al. ${ }^{[4]}$ published an interesting study in 2019. They focused on triple negative breast cancer which is characterized generally with poor prognosis and chemoresistance. Nanodiamonds used here were coated with polyglycerol and doxorubicin. Since immunosuppression plays an important role in that cancer, they looked at changes in the tumor environment and adjacent immunological parameters. The doxorubicin coated on nanodiamond carriers did not stimulate the upregulation of P-glycoprotein or interleukin-6, which both act as mediators of doxorubicin resistance. Importantly, the application of nanodiamondbased complexes resulted in the reduced secretion of the granulocyte-colony stimulating factor produced by the tumor and in the reduced production of myeloid-derived suppressor cells (MDSCs). The MDSC are myeloid cells reprogrammed by a tumor to suppress anti-tumor immune responses. This favorable environment led to the activation of macrophages, dendritic cells, and lymphocytes that effectively started an anti-tumor response ${ }^{[44]}$. That study is one of the best reports describing the immunological aspects of nanodiamond application so far. Such complex studies remain rare.

Recent remarkable or important studies have been completed mainly on breast and liver cancers, but there are individual reports describing similar findings in other cancers (e.g., colon, pancreas, lung, and leukemia) too. Tables 1 and 2 summarize exemplary and key studies describing resistance of cancer cells to conventional therapy. Table 1 focuses on studies performed in vitro and Table 2 involves studies performed in vivo [Tables 1 and 2]. So far, most of the reports suffer from incomplete characterization of 
Table 1. Summary of in vitro studies focused on drug - nanodiamond conjugates in order to aim cancer cells that are resistant to conventional treatment

\begin{tabular}{|c|c|c|c|c|}
\hline Author & Cancer/model & $\begin{array}{c}\text { ND core (size and } \\
\text { charge) }\end{array}$ & Conjugate/size & Conclusion \\
\hline Chow et al. ${ }^{[40]} 2011$ & Liver cancer (Huh7, LTM2) & $45 \mathrm{~nm}(\mathrm{DLS}) / 17 \mathrm{mV}$ & ND-DOX & $\begin{array}{l}\text { Decreased DOX efflux/tumor } \\
\text { regression }\end{array}$ \\
\hline Du et al. ${ }^{[76]} 2020$ & $\begin{array}{l}\text { Different solid tumors (HeLa, } \\
\text { HepG2, MCF-7, CHO) }\end{array}$ & $\begin{array}{l}166 \mathrm{~nm}(\mathrm{DLS} \\
\mathrm{SEM}) /-30 \mathrm{mV}\end{array}$ & $\begin{array}{l}\text { ND-PEG-HYD-FA- } \\
\text { DOX/264 nm, -19 mV } \\
\text { (DLS, SEM) }\end{array}$ & $\begin{array}{l}\text { Intracellular pH-activated drug } \\
\text { release, rapid accumulation in } \\
\text { cancer cells }\end{array}$ \\
\hline Lin et al. ${ }^{[41]} 2017$ & $\begin{array}{l}\text { Colon cancer (RKO, HCT116, } \\
\text { SW620) }\end{array}$ & $3-5 \mathrm{~nm}$ & ND-PTX, ND-PTX-Cet & $\begin{array}{l}\text { Induction of mitochondrial cell } \\
\text { death }\end{array}$ \\
\hline Wang et al. ${ }^{[43]} 2014$ & Liver cancer (LT2-MYC) & $11 \mathrm{~nm} / 48 \mathrm{mV}(\mathrm{DLS})$ & ND-EPI/89 nm & Prolonged drug retention \\
\hline Yuan et al. ${ }^{[44]} 2019$ & Breast cancer (4T1) & $5 \mathrm{~nm}(\mathrm{DLS})$ & DOX-PG-ND/84nm & $\begin{array}{l}\text { Reverses cancer-induced } \\
\text { immunosuppression }\end{array}$ \\
\hline Lam et al. ${ }^{[46]} 2018$ & $\begin{array}{l}\text { Lung cancer (A549, } \\
\mathrm{NCl}-\mathrm{H} 460, \mathrm{NCl}-\mathrm{H} 1975 \text { ) }\end{array}$ & $4 \mathrm{~nm} /-28 \mathrm{mV}(\mathrm{DLS})$ & $\begin{array}{l}\text { ND-GF-PEG, ND-EL- } \\
\text { PEG/94 nm, } 112 \text { nm }\end{array}$ & Decreased viability \\
\hline Zhang et al. ${ }^{[47]} 2011$ & $\begin{array}{l}\text { Breast cancer (MDA- } \\
\text { MB-231) }\end{array}$ & $50 \mathrm{~nm} / 15 \mathrm{mV}$ (DLS) & $\begin{array}{l}\text { FND-oligo } \\
\text {-PTX-antiEGFR }\end{array}$ & Specific cancer cell delivery \\
\hline $\begin{array}{l}\text { Madamsetty et al. }{ }^{[48]} \\
2019\end{array}$ & Pancreatic ductal carcinoma & $35 \mathrm{~nm}$ & $\begin{array}{l}\text { ND-PEG-DOX/76 nm, } \\
-10 \mathrm{mV} \text { (DLS) }\end{array}$ & $\begin{array}{l}\text { Increased drug efficacy and } \\
\text { lower side-effects }\end{array}$ \\
\hline Chan et al. ${ }^{[65]} 2017$ & $\begin{array}{l}\text { DOX-RS breast cancer (MCF- } \\
\text { 7) }\end{array}$ & $37 \mathrm{~nm} /-93 \mathrm{mV}$ & $\begin{array}{l}\text { FND-MLS-PeFA-DOX } \\
/ 279 \mathrm{~nm}\end{array}$ & $\begin{array}{l}\text { Targeting mitochondria, } \\
\text { increased DOX uptake }\end{array}$ \\
\hline Toh et al. ${ }^{[67]} 2014$ & $\begin{array}{l}\text { MTX-RS Breast cancer } \\
\text { (MDA-MB-231) }\end{array}$ & $23 \mathrm{~nm}(\mathrm{DLS}) / 56 \mathrm{mV}$ & ND-MTX & Enhanced drug reflux \\
\hline $\begin{array}{l}\text { Setyawati et al. }{ }^{[68]} \\
2016\end{array}$ & $\begin{array}{l}\text { Primary endothelial cells, } \\
\text { MDA-MB-468 }\end{array}$ & $5 \mathrm{~nm} /-24 \mathrm{mV}(\mathrm{TEM})$ & $\begin{array}{l}\text { ND and DOX, not } \\
\text { combined }\end{array}$ & $\begin{array}{l}\text { Increase of vascular permeability } \\
\text { in low-EPR tumors }\end{array}$ \\
\hline Man et al. ${ }^{[74]} 2014$ & DNR-RS leukemia (K562) & $51 \mathrm{~nm}(\mathrm{DLS})$ & ND-DNR/93 nm & Increased efficacy \\
\hline Zhang et al. ${ }^{[75]} 2014$ & Gastric cancer (BGC-823) & N/A & $\begin{array}{l}\text { (ND-SRF) liposom/128 } \\
\mathrm{nm}(\mathrm{DLS})\end{array}$ & $\begin{array}{l}\text { Improved drug bioavailability, } \\
\text { decreased tumor growth, } \\
\text { suppression of metastasis }\end{array}$ \\
\hline
\end{tabular}

Permanent cell lines were used. ND core is characterized with average size and overall surface charge. The method for size evaluation is stated if available. Cet: Cetuximab; FND: fluorescent nanodiamond; DLS: dynamic light scattering, hydrodynamic parameter; DNR: daunorubicin; DOX: doxorubicin; EPI: epirubicin; GF: gefitinib; EL: erlotinib; FA: folate; PeFA: PEGylated folic acid; HYD: hydrazine; PEG: polyethylenglycol; PG: polyglycerol; PTX: paclitaxel; MLS: mitochondrial localizing sequence peptide; MTX: Mitoxantrone; N/A: not available; ND: nanodiamond, unavailable method of ND preparation; RS: resistant; SRF: sorafenib

Table 2. Available studies in vivo that employed nanodiamonds to overcome cancer cell resistance

\begin{tabular}{|c|c|c|c|c|}
\hline Author & Cancer type & $\begin{array}{c}\text { ND core (size and } \\
\text { charge) }\end{array}$ & Conjugate/size & Conclusion \\
\hline Chow et al. ${ }^{[40]} 2011$ & Liver cancer (LTM2) & $45 \mathrm{~nm}(\mathrm{DLS}) / 17 \mathrm{mV}$ & ND-DOX & Decreased DOX efflux/tumor regression \\
\hline Du et al. ${ }^{[76]} 2020$ & $\begin{array}{l}\text { Liver cancer } \\
\text { (HepG2) }\end{array}$ & $\begin{array}{l}166 \mathrm{~nm}(\mathrm{DLS} \\
\mathrm{SEM}) /-30 \mathrm{mV}\end{array}$ & $\begin{array}{l}\text { ND-PEG-HYD-FA- } \\
\text { DOX/264 nm, -19 mV (DLS, } \\
\text { SEM) }\end{array}$ & $\begin{array}{l}\text { Specific accumulation in tumor, reduced } \\
\text { tumor growth, lower toxicity than free } \\
\text { DOX }\end{array}$ \\
\hline Lin et al. ${ }^{[41]} 2017$ & Colon cancer (RKO) & $3-5 \mathrm{~nm}$ & ND-PTX, ND-PTX-Cet & Reduced tumor size \\
\hline Moore et al. ${ }^{[42]} 2013$ & $\begin{array}{l}\text { Breast cancer } \\
\text { (MDA-MB-231) }\end{array}$ & $\begin{array}{l}60 \mathrm{~nm}(\mathrm{DLS}) / \text { near } \\
\text { neutral }\end{array}$ & $\begin{array}{l}\text { EGFR - (ND - epirubicin) } \\
\text { liposom }\end{array}$ & Complete tumor regression \\
\hline Wang et al. ${ }^{[43]} 2014$ & $\begin{array}{l}\text { Myc-induced liver } \\
\text { cancer }\end{array}$ & $11 \mathrm{~nm} / 48 \mathrm{mV}(\mathrm{DLS})$ & ND-EPI/89 nm & Prolonged drug retention \\
\hline Yuan et al. ${ }^{[44]} 2019$ & Breast cancer (4T1) & $5 \mathrm{~nm}(\mathrm{DLS})$ & DOX-PG-ND/84 nm & $\begin{array}{l}\text { Reverses cancer-induced } \\
\text { immunosuppression }\end{array}$ \\
\hline Zhang et al. ${ }^{[75]} 2014$ & $\begin{array}{l}\text { Gastric cancer } \\
\text { (BGC-823) }\end{array}$ & N/A & $\begin{array}{l}\text { (ND-SRF) liposom/128 nm } \\
\text { (DLS) }\end{array}$ & $\begin{array}{l}\text { Improved drug bioavailability, decreased } \\
\text { tumor growth, suppression of metastasis }\end{array}$ \\
\hline
\end{tabular}

ND core is characterized with average size and overall surface charge. Cet: Cetuximab; FND: fluorescent nanodiamond, HPHT preparation; DLS: dynamic light scattering, hydrodynamic parameter; DOX: doxorubicin; EGFR: epidermal growth factor receptor; EPI: epirubicin; FA: folate; HYD: hydrazine; PEG: polyethylenglycol; PG: polyglycerol; MTX: Mitoxantrone; N/A: not available; ND: nanodiamond, unavailable method of ND preparation; SRF: sorafenib

the nanomaterial or from suboptimal biological models. It is difficult to perform interdisciplinary research and fulfill ideally both physical chemistry and biology parameters. We have to accept that nanodiamonds represent a heterogeneous material, and each particle modification (size, shape, coating, sp2 amount, etc.) will have a specific impact on carrier behavior and final performance. Looking for an ideal formula 
(concerning desired use) would be easier if there was a united database containing the basic parameters (size, shape, source, preparation, surface charge) of nanodiamond cores as well as the coated constructs, employed biological model, and complex outputs. There is also a bit of concern using different methods for size evaluation. Most reports use hydrodynamic diameter (measured by dynamic light scattering) to characterize the coated nanoparticles; however, some coating combinations, might suffer from weak interaction among nanoparticles (due to macromolecule coating) or formation of larger clusters ${ }^{[69]}$. Then, atomic force microscopy could serve as an alternative method for size evaluation.

\section{Other carbon material against CSC}

Other carbon materials such as graphene oxide and carbon nanotubes were also used to overcome tumor chemoresistance, particularly by targeting the cancer stem cells. In the case of graphene oxide, Fiorillo et al. ${ }^{[70]}$ suggested that it targeted cancer stem cells based on their phenotype and inhibited several key signal pathways leading to the differentiation of cancer stem cells.

Carbon nanotubes are also a widely studied drug nanocarrier due to their unique properties like membrane penetrability, large drug loading, selective retention in the tumor, generally low toxicity, and photothermic properties. Their limitation for wider use is a thread of unpredictable increase in toxicity due to high impurities content, suboptimal production method, morphology, size, functionalization etc. ${ }^{[71]}$. Carbon nanotubes were considered in two main ways - thermal therapy and drug delivery. Burke et al. ${ }^{[72]}$ demonstrated that nanotube-mediated thermal therapy resulted in the impairment of stem cells renewal potential. The nanotubes were targeted against breast cancer stem cells via CD44 marker ${ }^{[72]}$. Yao et al. ${ }^{[73]}$ used another approach to eliminate cancer cells; they employed chitosan-coated single wall carbon nanotubes loaded with salinomycin and functionalized with hyaluronic acid.

\section{CONCLUSION}

Nanodiamonds have several unique properties that make them promising nanomaterial for biomedical applications. These include unique structure, electrostatic properties, a chemically inert core, and a tunable surface. Some nanodiamond properties enable them to challenge cancer cell drug-resistance by overcoming drug efflux, increasing feasible drug dosage while lowering side toxicity, and the effective targeting of cancer stem cells. There are numerous reports of successful in vitro and in vivo proof of concepts. Reports differ in applied drug-nanodiamond formulation or strategy, and any minor modification in coating or size affects final performance. It is impossible to make a general conclusion pointing out one superior strategy, but the variety of approaches holds great potential. It shows there are many possibilities to combine individual approaches, and tailor therapy to specific cancer type. Clearly, nanodiamond carriers shall remain in focus to accompany and improve conventional chemotherapeutics.

\section{DECLARATIONS}

\section{Authors' contributions}

Made substantial contributions to conception and design of the study: Benson V, Amini A

\section{Availability of data and materials}

Not applicable.

\section{Financial support and sponsorship}

None.

\section{Conflicts of interest}

All authors declared that there are no conflicts of interest. 


\section{Ethical approval and consent to participate}

Not applicable.

\section{Consent for publication}

Not applicable.

\section{Copyright}

(c) The Author(s) 2020.

\section{REFERENCES}

1. Alavi M, Hamidi M. Passive and active targeting in cancer therapy by liposomes and lipid nanoparticles. Drug Metab Pers Ther 2019;34: 20180032

2. Vladimis Y, Valerio V. Bringing again noble metal nanoparticles to the forefront of cancer therapy. Front Bioeng Biotechnol 2018;6:143.

3. Marchesan S, Kostarelos K, Bianco A, Prato M. The winding road for carbon nanotubes in nanomedicine. Mat Today 2015;18:12-9.

4. Tsai LW, Lin YC, Perevedentseva E, Lugovtsov A, Priezzhev A, et al. Nanodiamonds for medical applications: interaction with blood in vitro and in vivo. Int J Mol Sci 2016;17:1111.

5. Křivohlavá R, Neuhöferová E, Jakobsen KQ, Benson V. Knockdown of microRNA-135b in mammary carcinoma by targeted nanodiamonds: potentials and pitfalls of in vivo applications. Nanomaterials (Basel) 2019;9:866.

6. Suarez-Kelly LP, Campbell AR, Rampersaud IV, Bumb A, Wang MS, et al. Fluorescent nanodiamonds engage innate immune effector cells: a potential vehicle for targeted anti-tumor immunotherapy. Nanomedicine 2017;13:909-20.

7. Barnard AS. Diamond standard in diagnostics: nanodiamond biolabels make their mark. Analyst 2009;134:1751-64.

8. Faklaris O, Joshi V, Irinopoulou T, Tauc P, Sennour M, et al. Photoluminescent diamond nanoparticles for cell labeling: study of the uptake mechanism in mammalian cells. ACS Nano 2009;3:3955-62.

9. Hui YY, Cheng CL, Chang HC. Nanodiamonds for optical bioimaging. J Phys D Appl Phys 2010;43:374021.

10. Lukowski S, Neuhoferova E, Kinderman M, Krivohlava R, Mineva A, et al. Fluorescent nanodiamonds are efficient, easy-to-use cytocompatible vehicles for monitored delivery of non-coding regulatory RNAs. J Biomed Nanotechnol 2018;14:946-58.

11. McGuinness LP, Yan Y, Stacey A, Simpson DA, Hall LT, et al. Quantum measurement and orientation tracking of fluorescent nanodiamonds inside living cells. Nat Nanotechnol 2011;6:358-63.

12. Mochalin VN, Shenderova O, Ho D, Gogotsi Y. The properties and applications of nanodiamonds. Nat Nanotechnol 2011;7:11-23.

13. Petrakova V, Benson V, Buncek M, Fiserova A, Ledvina M, et al. Imaging of transfection and intracellular release of intact, non-labeled DNA using fluorescent nanodiamonds. Nanoscale 2016;8:12002-12.

14. Kaur R, Badea I. Nanodiamonds as novel nanomaterials for biomedical applications: drug delivery and imaging systems. Int J Nanomedicine 2013;8:203-20.

15. Turcheniuk K, Mochalin VN. Biomedical applications of nanodiamond (Review). Nanotechnology 2017;28:252001

16. Chang YR, Lee HY, Chen K, Chang CC, Tsai DS, et al. Mass production and dynamic imaging of fluorescent nanodiamonds. Nat Nanotechnol 2008;3:284-8.

17. Longmire M, Choyke PL, Kobayashi H. Clearance properties of nano-sized particles and molecules as imaging agents: considerations and caveats. Nanomedicine (Lond) 2008;3:703-17.

18. Fang J, Nakamura H, Maeda H. The EPR effect: unique features of tumor blood vessels for drug delivery, factors involved, and limitations and augmentation of the effect. Adv Drug Deliv Rev 2011;63:136-51.

19. Yu M, Zheng J. Clearance pathways and tumor targeting of imaging nanoparticles. ACS Nano 2015;9:6655-74.

20. Mohan N, Chen CS, Hsieh HH, Wu YC, Chang HC. In vivo imaging and toxicity assessments of fluorescent nanodiamonds in Caenorhabditis elegans. Nano Lett 2010;10:3692-9.

21. Vaijayanthimala V, Cheng PY, Yeh SH, Liu KK, Hsiao CH, et al. The long-term stability and biocompatibility of fluorescent nanodiamond as an in vivo contrast agent. Biomaterials 2012;33:7794-802.

22. Zheng T, Perona Martínez F, Storm IM, Rombouts W, Sprakel J, et al. Recombinant protein polymers for colloidal stabilization and improvement of cellular uptake of diamond nanosensors. Anal Chem 2017;89:12812-20.

23. Hemelaar SR, Nagl A, Bigot F, Rodríguez-García MM, de Vries MP, et al. The interaction of fluorescent nanodiamond probes with cellular media. Mikrochim Acta 2017;184:1001-9.

24. Daniels TR, Bernabeu E, Rodríguez JA, Patel S, Kozman M, et al. The transferrin receptor and the targeted delivery of therapeutic agents against cancer. Biochim Biophys Acta 2012;1820:291-317.

25. Chang LY, Osawa E, Barnard AS. Confirmation of the electrostatic self-assembly of nanodiamonds Nanoscale. 2011;3:958-62.

26. Chow EK. Implication of cancer stem cells in cancer drug development and drug delivery. J Lab Autom 2013;18:6-11

27. Chu Z, Zhang S, Zhang B, Zhang C, Fang CY, et al. Unambiguous observation of shape effects on cellular fate of nanoparticles. Sci Rep 2014;4:4495.

28. Puzyr A, Baron A, Purtov K, Bortnikov E, Skobelev N, et al. Nanodiamonds with novel properties: a biological study. Diam Relat Mater $2007 ; 16: 5$

29. van der Laan K, Hasani M, Zheng T, Schirhagl R. Nanodiamonds for in vivo applications. Small 2018;14:e1703838 
30. Hsiao WW, Hui YY, Tsai PC, Chang HC. Fluorescent nanodiamond: a versatile tool for long-term cell tracking, super-resolution imaging, and nanoscale temperature sensing. Acc Chem Res 2016;49:400-7.

31. Gulka M, Salehi H, Varga B, Middendorp E, Pall O, et al. Simultaneous label-free live imaging of cell nucleus and luminescent nanodiamonds. Sci Rep 2020;10:9791.

32. Gismondi A, Reina G, Orlanducci S, Mizzoni F, Gay S, et al. Nanodiamonds coupled with plant bioactive metabolites: a nanotech approach for cancer therapy. Biomaterials 2015;38:22-35.

33. Martín R, Alvaro M, Herance JR, García H. Fenton-treated functionalized diamond nanoparticles as gene delivery system. ACS Nano 2010;4:65-74.

34. Chen M, Zhang XQ, Man HB, Lam R, Chow EK, et al. Nanodiamond vectors functionalized with polyethylenimine for siRNA delivery. J Phys Chem Lett 2010;1:3167-71.

35. Ho D, Wang CH, Chow EK. Nanodiamonds: the intersection of nanotechnology, drug development, and personalized medicine. Sci Adv 2015;1:e1500439.

36. Lee DK, Kee T, Liang Z, Hsiou D, Miya D, et al. Clinical validation of a nanodiamond-embedded thermoplastic biomaterial. Proc Natl Acad Sci U S A 2017;114:E9445-54.

37. Nakamura Y, Mochida A, Choyke PL, Kobayashi H. Nanodrug delivery: is the enhanced permeability and retention effect sufficient for curing cancer? Bioconjug Chem 2016;27:2225-38.

38. Ramzy L, Nasr M, Metwally AA, Awad GAS. Cancer nanotheranostics: a review of the role of conjugated ligands for overexpressed receptors. Eur J Pharm Sci 2017;104:273-92.

39. Cersosimo RJ, Hong WK. Epirubicin: a review of the pharmacology, clinical activity, and adverse effects of an adriamycin analogue. J Clin Oncol 1986;4:425-39.

40. Chow EK, Zhang XQ, Chen M, Lam R, Robinson E, et al. Nanodiamond therapeutic delivery agents mediate enhanced chemoresistant tumor treatment. Sci Transl Med 2011;3:73ra21.

41. Lin YW, Raj EN, Liao WS, Lin J, Liu KK, et al. Co-delivery of paclitaxel and cetuximab by nanodiamond enhances mitotic catastrophe and tumor inhibition. Sci Rep 2017;7:9814.

42. Moore LK, Chow EK, Osawa E, Bishop JM, Ho D. Diamond-lipid hybrids enhance chemotherapeutic tolerance and mediate tumor regression. Adv Mater 2013;25:3532-41.

43. Wang X, Low XC, Hou W, Abdullah LN, Toh TB, et al. Epirubicin-adsorbed nanodiamonds kill chemoresistant hepatic cancer stem cells. ACS Nano 2014;8:12151-66.

44. Yuan SJ, Xu YH, Wang C, An HC, Xu HZ, et al. Doxorubicin-polyglycerol-nanodiamond conjugate is a cytostatic agent that evades chemoresistance and reverses cancer-induced immunosuppression in triple-negative breast cancer. J Nanobiotechnology 2019;17:110.

45. Huang H, Pierstorff E, Osawa E, Ho D. Active nanodiamond hydrogels for chemotherapeutic delivery. Nano Lett 2007;7:3305-14.

46. Lam R, Chen M, Pierstorff E, Huang H, Osawa E, et al. Nanodiamond-embedded microfilm devices for localized chemotherapeutic elution. ACS Nano 2008;2:2095-102.

47. Zhang XQ, Lam R, Xu X, Chow EK, Kim HJ, et al. Multimodal nanodiamond drug delivery carriers for selective targeting, imaging, and enhanced chemotherapeutic efficacy. Adv Mater 2011;23:4770-5.

48. Madamsetty VS, Sharma A, Toma M, Samaniego S, Gallud A, et al. Tumor selective uptake of drug-nanodiamond complexes improves therapeutic outcome in pancreatic cancer. Nanomedicine 2019;18:112-21.

49. Hoang Thi TT, Pilkington EH, Nguyen DH, Lee JS, Park KD, et al. The importance of Poly(ethylene glycol) alternatives for overcoming PEG immunogenicity in drug delivery and bioconjugation. Polymers (Basel) 2020;12:298.

50. Suliman S, Xing Z, Wu X, Xue Y, Pedersen TO, et al. Release and bioactivity of bone morphogenetic protein-2 are affected by scaffold binding techniques in vitro and in vivo. J Control Release 2015;197:148-57.

51. Manus LM, Mastarone DJ, Waters EA, Zhang XQ, Schultz-Sikma EA, et al. Gd(III)-nanodiamond conjugates for MRI contrast enhancement. Nano Lett 2010;10:484-9.

52. Wu TJ, Tzeng YK, Chang WW, Cheng CA, Kuo Y, et al. Tracking the engraftment and regenerative capabilities of transplanted lung stem cells using fluorescent nanodiamonds. Nat Nanotechnol 2013;8:682-9.

53. Lyakhovich A, Lleonart ME. Bypassing mechanisms of mitochondria-mediated cancer stem cells resistance to chemo- and radiotherapy. Oxid Med Cell Longev 2016;2016:1716341.

54. Li L, Bhatia R. Stem cell quiescence. Clin Cancer Res 2011;17:4936-41.

55. Saha S, Adhikary A, Bhattacharyya P, Das T, Sa G. Death by design: where curcumin sensitizes drug-resistant tumours. Anticancer Res 2012;32:2567-84.

56. Sotiropoulou PA, Christodoulou MS, Silvani A, Herold-Mende C, Passarella D. Chemical approaches to targeting drug resistance in cancer stem cells. Drug Discov Today 2014;19:1547-62.

57. Vinogradov S, Wei X. Cancer stem cells and drug resistance: the potential of nanomedicine. Nanomedicine (Lond) 2012;7:597-615.

58. Hill C, Wang Y. The importance of epithelial-mesenchymal transition and autophagy in cancer drug resistance. Cancer Drug Resist 2020;3:38-47.

59. Wang Y, Hays E, Rama M, Bonavida B. Cell-mediated immune resistance in cancer. Cancer Drug Resist 2020;3:232-51.

60. Sun Z, Zhao Z, Li G, Dong S, Huang Z, et al. Relevance of two genes in the multidrug resistance of hepatocellular carcinoma: in vivo and clinical studies. Tumori 2010;96:90-6.

61. Qin W, Huang G, Chen Z, Zhang Y. Nanomaterials in targeting cancer stem cells for cancer therapy. Front Pharmacol 2017;8:1.

62. Stupp R, Hegi ME. Targeting brain-tumor stem cells. Nat Biotechnol 2007;25:193-4. 
63. Hong IS, Jang GB, Lee HY, Nam JS. Targeting cancer stem cells by using the nanoparticles. Int J Nanomedicine 2015;10:251-60.

64. Ali MS, Metwally AA, Fahmy RH, Osman R. Nanodiamonds: minuscule gems that ferry antineoplastic drugs to resistant tumors. Int J Pharm 2019;558:165-76.

65. Chan MS, Liu LS, Leung HM, Lo PK. Cancer-cell-specific mitochondria-targeted drug delivery by dual-ligand-functionalized nanodiamonds circumvent drug resistance. ACS Appl Mater Interfaces 2017;9:11780-9.

66. Batist G, Ramakrishnan G, Rao CS, Chandrasekharan A, Gutheil J, et al. Reduced cardiotoxicity and preserved antitumor efficacy of liposome-encapsulated doxorubicin and cyclophosphamide compared with conventional doxorubicin and cyclophosphamide in a randomized, multicenter trial of metastatic breast cancer. J Clin Oncol 2001;19:1444-54.

67. Toh TB, Lee DK, Hou W, Abdullah LN, Nguyen J, et al. Nanodiamond-mitoxantrone complexes enhance drug retention in chemoresistant breast cancer cells. Mol Pharm 2014;11:2683-91.

68. Setyawati MI, Mochalin VN, Leong DT. Tuning endothelial permeability with functionalized nanodiamonds. ACS Nano 2016;10:1170-81.

69. Hoo CM, Starostin N, West P, Mecartney ML. A comparison of atomic force microscopy (AFM) and dynamic light scattering (DLS) methods to characterize nanoparticle size distributions. J Nanopart Res 2008;10:89-96.

70. Fiorillo M, Verre AF, Iliut M, Peiris-Pagés M, Ozsvari B, et al. Graphene oxide selectively targets cancer stem cells, across multiple tumor types: implications for non-toxic cancer treatment, via "differentiation-based nano-therapy". Oncotarget 2015;6:3553-62.

71. Alshehri R, Ilyas AM, Hasan A, Arnaout A, Ahmed F, et al. Carbon nanotubes in biomedical applications: factors, mechanisms, and remedies of toxicity. J Med Chem 2016;59:8149-67.

72. Burke AR, Singh RN, Carroll DL, Torti FM, Torti SV. Targeting cancer stem cells with nanoparticle-enabled therapies. J Mol Biomark Diagn 2012; doi: 10.4172/2155-9929.S8-003.

73. Yao HJ, Zhang YG, Sun L, Liu Y. The effect of hyaluronic acid functionalized carbon nanotubes loaded with salinomycin on gastric cancer stem cells. Biomaterials 2014;35:9208-23.

74. Man HB, Kim H, Kim HJ, Robinson E, Liu WK, et al. Synthesis of nanodiamond-daunorubicin conjugates to overcome multidrug chemoresistance in leukemia. Nanomedicine 2014;10:359-69.

75. Zhang Z, Niu B, Chen J, He X, Bao X, et al. The use of lipid-coated nanodiamond to improve bioavailability and efficacy of sorafenib in resisting metastasis of gastric cancer. Biomaterials 2014;35:4565-72.

76. Du X, Li L, Wei S, Wang S, Li Y. A tumor-targeted, intracellular activatable and theranostic nanodiamond drug platform for strongly enhanced in vivo antitumor therapy. J Mater Chem B 2020;8:1660. 